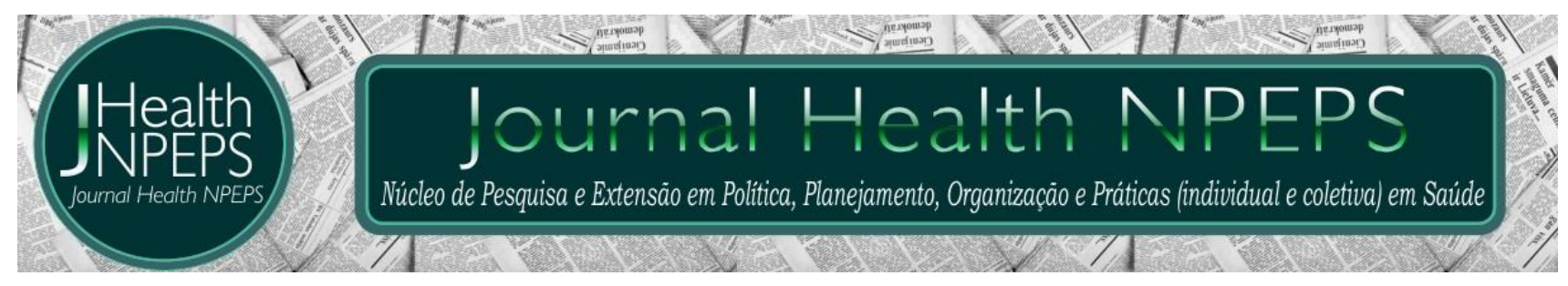

http://dx.doi.org/10.30681/252610104440

ARTIGO ORIGINAL

\title{
Independência funcional e o estado confusional de pessoas sujeitas a programa de reabilitação
}

\section{Functional Independence of people presenting confusional state subject to rehabilitation}

\section{Independencia functional y el estado de confusión de las personas sujetas a programa de rehabilitación}

\section{Andreia Maria Lima1 ${ }^{1}$, Maria Salomé Martins Ferreira ${ }^{2}$, Maria Manuela Ferreira Pereira da Silva Martins ${ }^{3}$, Carla Silvia Fernandes ${ }^{4}$, Maria Teresa Ferreira Moreira ${ }^{5}$, Tânia Marisa Pinto Rodrigues ${ }^{6}$}

\section{RESUMO}

Objetivo: conhecer a influência do estado confusional na recuperação da independência funcional do paciente sujeito a reabilitação. Método: estudo descritivo-correlacional, quantitativo e longitudinal, com amostragem não probabilística, de tipologia acidental. Critérios de inclusão: medida de independência funcional $\leq 90$ e pacientes sujeitos a imobilidade. Critérios de exclusão: pacientes portadores de doenças neoplásicas e com uma escala de coma de Glasgow <11. Para a coleta de dados, utilizou-se um questionário sociodemográfico e as escalas NEECHAM e MIF. Resultados: os dados da amostra $(n=40)$, revelam idade de 76,48 anos, em sua maioria mulheres, casadas, com o primeiro ciclo e em média estiveram sujeitos a 49,60 dias de imobilidade. 0 estado confusional teve uma melhoria significativa da $1^{\text {a }}$ avaliação na admissão, na qual $52,2 \%$ dos indivíduos apresentavam-se com confusão moderada a severa, para a $2^{a}$ avaliação na alta, em que $85 \%$ apresentavam-se não confusos. A independência funcional registou uma melhoria

\footnotetext{
${ }^{1}$ Enfermeira. Mestre em Enfermagem de Reabilitação. Professora Adjunta da Escola Superior de Saúde - Fernando Pessoa. Porto, Portugal. E-mail: alima2358@hotmail.com ORCID ID: https://orcid.org/0000-0001-7535-9040 Autor para correspondência - Endereço: Rua da Travessa, n.235, 4905-650 Vila de Punhe, Portugal.

${ }^{2}$ Enfermeira. Doutora em Psicologia da Saúde. Vice-Presidente da Escola Superior de Saúde do Instituto Politécnico de Viana do Castelo. Viana do Castelo, Portugal. E-mail: salomeferreira@ess.ipvc.pt ORCID ID: https://orcid.org/00000003-1685-9891

${ }^{3}$ Enfermeira. Doutora em Ciências de Enfermagem. Professora Coordenadora na Escola Superior de Enfermagem do Porto. Porto, Portugal. E-mail: mmartins@esenf.pt ORCID ID: https://orcid.org/0000-0003-1527-9940

${ }^{4}$ Enfermeira. Doutora em Enfermagem. Professora Adjunta da Escola Superior de Enfermagem do Porto. Porto, Portugal. E-mail: carlasilvaf@gmail.com ORCID ID: https://orcid.org/0000-0001-7251-5829

${ }^{5}$ Enfermeira. Doutora em Enfermagem. Professora Adjunta da Escola Superior de Saúde - Fernando Pessoa. Porto, Portugal. E-mail: tmoreira@ufp.edu.pt ORCID ID: https://orcid.org/0000-0002-2554-697X

${ }^{6}$ Enfermeira. Mestre em Enfermagem de Reabilitação. Enfermeira Especialista em Enfermagem de Reabilitação no Centro Hospitalar São João. Porto, Portugal. E-mail: tmarisaprodrigues@gmail.com ORCID ID: https://orcid.org/0000$\underline{0003-4785-3783}$
}

Este artigo está licenciado sob forma de uma licença Creative Commons Atribuição 4.0 Internacional, que permite uso irrestrito, distribuição e reprodução em qualquer meio, desde que a publicação original seja corretamente citada 
de 38,03\%. Conclusão: a confusão é um problema neuropsiquiátrico que interfere na atenção e a cognição, nomeadamente na capacidade de percepção. No entanto, mesmo os participantes confusos, quando submetidos a um programa de enfermagem de reabilitação tendem a recuperar a independência funcional.

Descritores: Enfermagem; Reabilitação; Independência; Confusão; Pessoas Acamadas.

\section{ABSTRACT}

Objective: to know the influence of the confusional state in the recovery of the functional independence of the patient undergoing rehabilitation. Method: descriptivecorrelational, quantitative and cross-sectional study, with non-probabilistic sampling, of accidental typology. Inclusion criteria: measure of functional independence $\leq 90$ and patients subject to immobility. Exclusion criteria: patients with neoplastic diseases and with a Glasgow coma scale $<11$. For data collection, a sociodemographic questionnaire and the NEECHAM and MIF scales were used. Results: the sample data $(n=40)$, reveal an age of 76.48 years, mostly women, married, with the elementary school and on average were subject to 49.60 days of immobility. The confusional state had a significant improvement from the 1st assessment on admission, in which $52.2 \%$ of the individuals presented with moderate to severe confusion, for the 2nd assessment at discharge, in which $85 \%$ were not confused. Functional independence improved by $38.03 \%$. Conclusion: confusion is a neuropsychiatric problem that interferes with attention and cognition, namely the capacity for perception. However, even confused participants, when submitted to a rehabilitation nursing program, tend to regain functional independence.

Descriptors: Nursing; Rehabilitation; Independence; Confusion; Bedridden Person.

\section{RESUMEN}

Objetivo: conocer la influencia del estado de confusión en la recuperación de la independencia funcional del paciente sometido a rehabilitación. Método: estudio descriptivo-correlacional, cuantitativo y transversal, con muestreo no probabilístico, de tipología accidental. Criterios de inclusión: medida de independencia funcional $\leq 90$ y pacientes sujetos a inmovilidad. Criterios de exclusión: pacientes con enfermedades neoplásicas y con una escala de coma de Glasgow <11. Para la recolección de datos, se utilizó un cuestionario sociodemográfico y las escalas NEECHAM y MIF. Resultados: los datos de la muestra $(n=40)$ revelan una edad de 76,48 años, en su mayoría mujeres, casadas, con el primer ciclo y en promedio estuvieron sujetas a 49,60 días de inmovilidad. El estado de confusión tuvo una mejoría significativa desde la primera evaluación (52.5\%) al ingreso, donde los individuos presentaron confusión de moderada a severa, para la segunda evaluación, día de alta, en la que el $85 \%$ no estaba confundido. La independencia funcional mejoró en 38.03\%. Conclusión: La confusión es un problema neuropsiquiátrico que interfiere con la atención y la cognición, es decir, la capacidad de percepción. Sin embargo, incluso los participantes confundidos, cuando se someten a un programa de rehabilitación de enfermería, tienden a recuperar la independencia funcional.

Descriptores: Enfermería; Rehabilitación; Independencia; Confusión; Personas Encamadas. 
imobilidade, na medida em que acarreta graves problemas para a saúde dos indivíduos. As pessoas recorrem com regularidade à realização do exercício físico e no caso dos idosos esta atividade assume, ainda maior importância ${ }^{1,2}$.

Inerente à importância da redução da morbilidade e comorbilidade, nos idosos salienta-se a necessidade da adoção de comportamentos saudáveis, sendo estes determinantes na diminuição do seu risco ${ }^{3}$, na linha da prevenção primordial e primária. Numa vertente secundária e terciária da prevenção surgem os programas de reabilitação, na medida em que estes reduzem não só os défices instalados, mas previnem outros problemas que decorrem da falta de mobilidade ${ }^{1}$.

A população portuguesa com mais de 65 anos, segundo o Instituto Nacional de Estatística, tem aumentado consideravelmente nos últimos anos, sendo, portanto, o envelhecimento um fenómeno social crescente. Acredita-se que o agravamento do envelhecimento demográfico, em Portugal, vai continuar e tenderá a estabilizar apenas daqui a 40 anos, referenciando-se o ano de $2060^{4}$.

o envelhecimento individual é um processo biopsicossocial, que depende de múltiplos fatores, nomeadamente biológicos, sociais, económicos, ambientais e históricos ${ }^{5}$, em geral, o declínio tem inicio durante a terceira década de vida, e acelera a partir dos 60 anos, especialmente: ao nível da taxa metabólica basal, o total de água no organismo, o débito cardíaco em repouso, a perfusão renal e a capacidade pulmonar total ${ }^{6}$, e muito do decréscimo destas funções pode estar relacionado com o descondicionamento e o desuso, sendo, portanto, o idoso das pessoas, o mais propenso a apresentar lesões patológicas nos mais diversos sistemas orgânicos ${ }^{7}$.

Associado ao envelhecimento, surge o compromisso do desempenho neuromuscular, manifestado pela fraqueza muscular, pela lentificação dos movimentos, pela perda da massa e força muscular e pela fadiga muscular precoce $^{8,9}$.

A perda ou diminuição da capacidade de função do idoso, como consequência de doenças físicas ou mentais, pode culminar na necessidade de adoção de estratégias e intervenções que visem a manutenção da independência e autonomia da pessoa, as quais requerem um trabalho constante dos profissionais que atuam, principalmente, ao nível da prevenção primordial e primária da saúde ${ }^{10}$. 
O estado confusional nos idosos é muito comum, sendo uma experiência desafiante para os prestadores de cuidados informais e mesmo os cuidadores formais. Estes quadros são altamente incidentes, nos idosos internados, constituindo um fator desgastante para os profissionais de saúde, devido às dificuldades que enfrentam quando pretendem prevenir, nomeadamente quedas e outros acidentes, implementar um tratamento ${ }^{11}$ e obter o consentimento informado, assim, como no momento da implementação um programa de reabilitação.

As causas que motivam a perda ou diminuição da função do idoso, podem ser de ordem: física, emocional, intelectual e social. Como resultado destas alterações, associadas às comorbilidades, surgem as consequências da própria imobilidade. Mesmo nas pessoas saudáveis, a imobilidade acarreta graves consequências ao nível dos vários sistemas orgânicos, designadamente nos sistemas: respiratório, cardiovascular, gastrointestinal, urinário, metabólico, sistema nervoso, pele e tegumentos e músculo-esquelético ${ }^{12}$.

O contexto ambiental, social e económico e a participação da familia, assumem aqui uma maior dimensão, influenciando a funcionalidade e o programa de reabilitação na sua conceção, na medida em que este programa deverá adequar-se às particularidades da pessoa ${ }^{13}$. Estas particularidades resultam das múltiplas patologias de que é portador, à polimedicação, à diminuição da funcionalidade, inerente ao próprio envelhecimento, às alterações sensoriais, cognitivas e nutricionais ${ }^{13}$.

Assim, a reabilitação é reconhecida, como uma componente fundamental e essencial no processo saúde-doença, a todos os níveis de vida, especialmente na melhoria da capacidade física e cognitiva, do ser humano ${ }^{14,15}$.

0 enfermeiro especialista em cuidados de reabilitação, concebe, implementa e monitoriza planos de enfermagem de reabilitação diferenciados, baseados nos problemas e potenciais das pessoas. O seu papel é crucial em todo o ciclo vital, na promoção de ações orientadas para a prevenção e tratamento da doença e para a promoção dos processos de readaptação/reintegração da pessoa, objetivando a manutenção das suas capacidades funcionais e cognitivas e optimizar a qualidade de vida das 
pessoas portadoras de doença ou deficiência, da família e comunidade, facilitando a sua socialização e fundamentalmente a sua dignidade e unicidade ${ }^{16,17}$.

O envelhecimento da população é um dado irrefutável e com ele surgem novas problemáticas relacionadas com a perda da funcionalidade e da cognição, com consequências a vários níveis da vida da pessoa. 0 contributo da enfermagem de reabilitação, nomeadamente na implementação de programas de intervenção especializada é inegável, uma vez que esta permite à pessoa restabelecer a sua autonomia, diminui consideravelmente a necessidade de recursos aos cuidados de saúde, diminui os reinternamentos, diminui a média de dias de internamento e os custos associados, diminui a incidência e prevalência de problemas como as úlceras de pressão, as infeções respiratórias, entre outras, e colabora ativamente na reintegração social.

Para a implementação de um programa de reabilitação ajustado tornase fundamental, o diagnóstico cognitivo e funcional, sendo, portanto, necessário recorrer a instrumentos de reflitam essas necessidades.

Com a certeza da importância do assunto em estudo, pretende-se dar resposta às seguintes questões de investigação: Qual o padrão funcional do paciente sujeito a imobilidade? e será que o estado confusional, influência a recuperação da independência funcional do paciente sujeito a reabilitação?, com o objetivo de conhecer a influência do estado confusional na recuperação da independência funcional do paciente sujeito a reabilitação.

\section{METODO}

Estudo descritivo-correlacional e longitudinal, sustentado numa estratégia de metodologia de investigação de natureza quantitativa e uma amostragem não probabilista de tipologia acidental.

Os participantes foram selecionados, independentemente do diagnóstico ou motivo de internamento e dos antecedentes pessoais, mas que apresentassem uma Medida de Independência Funcional (MIF) $\leq 90$ e estivessem sujeitos a imobilidade. Foram excluídos os portadores de doenças neoplásicas e com alterações do estado de consciência, com uma Escala de Coma de Glasgow $<11$.

De acordo com as normas de Helsínquia, após o parecer favorável da instituição, as pessoas envolvidas no estudo ou os seus substitutos legais, 
deram o seu consentimento, onde expressaram a liberdade de escolha na participação no estudo, sendo-lhes garantida a confidencialidade, privacidade e anonimato, tal como se pretende nos estudos de investigação.

A coleta de dados foi efetuada no período de Setembro de 2013 a Janeiro de 2014. Após a admissão dos pacientes no serviço de medicina interna, de um hospital de medio porte a norte de Portugal. Este hospital possui cerca de 180 leitos, dois blocos operatórios, uma unidade de cuidados intensivos e os serviços de medicina interna, cirurgia, pediatria, obstétricia, ginecologia, ortopedia, gastroenteriologia, unidade de AVC, unidade de dia e consultas externas. Foram identificados 62 participantes em potencial, porém somente 40 atenderam a todos os critérios de elegibilidade.

A coleta de dados foi efetuada em dois momentos diferentes. $A 1^{\text {a }}$ avaliação foi realizada no primeiro contato entre o participantes e o enfermeiro de reabilitação. A $2^{\mathrm{a}}$ avaliação, foi realizada no último dia de contato do participante com 0 enfermeiro de reabilitação, antes da alta. Durante todo o internamento, em uma média de 11 dias, os participantes foram submetidos a um programa de reabilitação de acordo com as suas necessidades, onde foram implementadas intervenções, para promover as capacidades físicas e cognitivas dos participantes. Durante o período de internamento, o enfermeiro especialista em cuidados de enfermagem de reabilitação, prestou cuidados de reabilitação a estes pacientes, todos os dias, de acordo com o plano de cuidados. Este plano foi elaborado com base nas necessidades de cada um, podendo variar desde exercícios musculares e articulares ativos, ativos resistidos, ativos assistidos e passivos, reeduação postural e tarefas de autocuidados, técnicas de cinesiterapia respiratória, treino de equilíbrio, treino de marcha, rolamento, ponte, capacitação cognitiva, através de livro de imagens e jogos de memória, conforme Quadro 1.

Foi aplicado um questionário sociodemográfico, a escala de Neelon and Champagne Confusion Scale $\left(\right.$ NEECHAM) ${ }^{18}$ e a MIF $^{19}$, na primeira (dia de admissão do paciente) e segunda avaliação (dia da alta do doente). O questionário sociodemográfico possibilitou o levantamento de informações acerca das caraterísticas sociodemográficas e clínicas dos participantes. Este questionário incluiu 
os seguintes itens: idade, nível de escolaridade, sexo, estado civil, proveniência, existência ou não de prestador de cuidados, tempo de imobilidade, diagnóstico de internamento, antecedentes pessoais $\mathrm{e}$ destino no momento da alta.

A NEECHAM Confusion Scale é uma escala que se baseia na observação e aprecia nove itens, dividindo-se em três subescalas: processamento, comportamento e controle fisiológico. Esta requer a avaliação dos sinais vitais, nomeadamente pressão arterial, frequência cardíaca, frequência respiratória e temperatura corporal, e avaliação da oximetria de pulso para ser possível a sua quantificação. Estes dados apesar de serem recolhidos diariamente de oito em oito horas aos pacientes, na amostra foram também avaliados para preenchimento da escala, na admissão e no momento da alta. Os scores variam entre zero (resposta mínima) a 30 (função normal), em que os valores são avaliados entre os parâmetros: <=19, sendo considerado um estado confusional agudo moderado a grave; de 20 a 24, encontram-se moderadamente confusos; 25 a 26, apresentam algum risco para desenvolver confusão; e de 27 a 30 , estado cognitivo normal ${ }^{18}$.

A MIF é uma escala que avalia a capacidade do indivíduo na realização de um conjunto de 18 tarefas, referentes a seis subescalas: Auto-cuidado que é dividida em seis itens (Alimentação; Higiene Pessoal; Banho; Vestir metade superior; Vestir metade inferior; Utilização do vaso sanitário), Controle de Esfíncteres em dois itens (Controle de urina; Controle das fezes), Mobilidade transferências em três itens (Leito, cadeira, cadeira de rodas; Vaso sanitário; Banheira, chuveiro), Locomoção em dois itens (Larcha/cadeira de rodas; Escadas), Comunicação em dois itens (Compreensão; Expressão) e Cognição Social em três itens (Interação social; Resolução de problemas; Memória). A pontuação de cada item varia de um a sete, de acordo com o grau de dependência: sete corresponde a independência completa, seis a independência modificada, cinco à necessidade de supervisão durante a realização da tarefa, quatro a ajuda mínima, ou seja o paciente realiza mais de $75 \%$ da tarefa, três a ajuda moderada, sendo que o paciente realiza mais de $50 \%$ da tarefa, dois a ajuda máxima, em que o paciente realiza mais de $25 \%$ da tarefa, um a ajuda total, ou seja o paciente tem que ser substituído em toda a tarefa. A MIF total pode ser 
dividida em quatro subescores, consoante a pontuação total obtida: 18 pontos (dependência completa); 19 - 60 pontos (dependência modificada, tendo assistência até 50\% da tarefa); 61 - 103 pontos (dependência modificada, tendo assistência até 25\% da tarefa); $104-12$ pontos (independência completa) ${ }^{19}$.

Os dados foram tratados através do programa estatístico Statistical Package Social Sciences (SPSS), versão 22.0, recorrendo à análise descritiva e inferencial. Foi aplicado o teste ShapiroWilk para avaliar a normalidade de distribuição, teste $\mathrm{t}$ de Student para amostras emparelhadas para testar a hipótese e o teste de correlação de Pearson para correlacionar as variáveis das escalas, uma vez que estas apresentam normalidade de distribuição. Para testar a hipótese optou-se pela aplicação do teste de Spearman, uma vez que as variáveis não apresentaram distribuição normal.

$\mathrm{Na}$ versão do estudo, a NEECHAM e a MIF apresentaram um Alfa de Cronbach de 0.870 e 0.964, respectivamente, o que exprime um bom índice de fidelidade.

Relativamente às variaveis em estudo, foi assumido como variável dependente, a independência funcional e o estado confusional, e como variável independente, os cuidados de enfermagem de reabilitação.

Quadro 1 - Programa de reabilitação implementado. Viana do Castelo, Portugal.

\begin{tabular}{lc}
\multicolumn{1}{c}{ Intervenções de enfermagem de reabilitação } & Frequência \\
\hline Técnicas de cinesiterapia respiratória & $1 \mathrm{vez} / \mathrm{dia}$ \\
Exercícios musculares passivos, ativos assistidos, ativos e ativos resistidos dos quatro & $1 \mathrm{vez} / \mathrm{dia}$ \\
membros & $1 \mathrm{vez} / \mathrm{dia}$ \\
Reeducação postural e tarefas de autocuidados & $1 \mathrm{vez} / \mathrm{dia}$ \\
Treino de equilíbrio & $1 \mathrm{Vez} / \mathrm{dia}$ \\
Exercicios de rolamento e ponte & $1 \mathrm{vez} / \mathrm{dia}$ \\
Treino de marcha & $2 \mathrm{vezes} / \mathrm{dia}$ \\
Exercícios de capacitação cognitiva & \\
\hline
\end{tabular}

\section{RESULTADOS E DISCUSSÃO}

A amostra apresentava uma média de idades de 76,48 anos, a maioria (70\%) com o $1^{\circ}$ ciclo, do sexo feminino $57,5 \%$ e com o estado civil de casados/união de fato (55\%). No que respeita à proveniência a grande maioria veio diretamente do seu domicílio/lar (90\%), enquanto que os restantes $10 \%$, foram transferidos de outro hospital, nomeadamente de uma unidade de cuidados intensivos.

Relativamente à existência de prestadores de cuidados, 57,5\% já apresentavam prestadores de cuidados, 
sendo eles amigos, assistentes confusional, na $1^{\text {a }}$ avaliação, $52,5 \%$ dos operacionais, esposa ou marido, filhos, participantes apresentavam confusão netos, sobrinhos e vizinhos.

Quanto aos motivos de internamento, apresentaram patologias de todos os tipos, no entanto, a maioria $(\mathrm{n}=26)$ correspondia ao sistema neurológico, seguido do sistema respiratório $(n=11)$ e dos sistemas cardíaco e metabólico $(n=8)$, com mesmo quantitativo. moderada a severa e na $2^{a}$ avaliação $85 \%$ apresentavam não confusos (Tabela 1).

Quanto a medida de independência funcional, verificou-se que em média, aumentou substancialmente da $1^{\mathrm{a}}$ para a $2^{\mathrm{a}}$ avaliação, o que significa que os participantes recuperaram a sua independência (Tabela 2).

Os participantes estiveram em média, sujeitos a 49,6 dias de imobilidade. No que concerne ao estado

\begin{tabular}{|c|c|c|c|c|}
\hline \multirow{4}{*}{$\begin{array}{l}\text { Escala de NEECHAM } \\
\text { Confusão moderada a severa } \\
\text { Confusão ligeira ou início de } \\
\text { desenvolvimento }\end{array}$} & \multicolumn{2}{|c|}{ 1ª Avaliação } & \multicolumn{2}{|c|}{$2^{\mathrm{a}}$ Avaliação } \\
\hline & $\mathrm{n}$ & $\%$ & $\mathbf{n}$ & $\%$ \\
\hline & 21 & 52.5 & 1 & 2.5 \\
\hline & 9 & 22.5 & 3 & 7.5 \\
\hline $\begin{array}{l}\text { Não confuso, mas com alto } \\
\text { risco }\end{array}$ & 5 & 12.5 & 2 & 5.0 \\
\hline Não confuso & 5 & 12.5 & 34 & 85.0 \\
\hline
\end{tabular}

Tabela 2 - Distribuição da amostra segundo a medida de independência funcional na $1 .^{\mathrm{a}}$ e na segundo avaliação, para cada subescala. Viana do Castelo, Portugal. $(n=40)$

\begin{tabular}{lcccc}
\hline \multirow{2}{*}{$\begin{array}{c}\text { MIF } \\
\text { (subescalas) }\end{array}$} & \multicolumn{2}{c}{$\mathbf{1}^{\text {a }}$ Avaliação } & \multicolumn{2}{c}{$\mathbf{2}^{\text {a }}$ Avaliação } \\
Autocuidados & Média & Dp & Média & Dp \\
\cline { 2 - 5 } Controle de esfíncteres & 12.5 & 5.724 & 30.35 & 6.833 \\
Mobilidade-transferências & 4.33 & 2.314 & 10.90 & 3.296 \\
Locomoção & 6.20 & 2.366 & 14.33 & 3.990 \\
Comunicação & 3.18 & 1.130 & 7.63 & 2.771 \\
Cognição social & 8.38 & 3.499 & 12.55 & 2.591 \\
TOTAL & 12.10 & 5.565 & 18.85 & 3.997 \\
\hline
\end{tabular}

Os resultados obtidos evidenciam diferenças no nível de independência funcional entre os dois momentos de avaliação, sendo estes, estatisticamente 
significativos $(p=0.000)$ para todas as subescalas da MIF. Relativamente à MIF, na $1^{\text {a }}$ avaliação observou-se que a média total era de 46,68 e que na $2^{a}$ avaliação passou para 94,60 . Isto demonstra que os participantes entre a $1^{\mathrm{a}}$ e a $2^{\mathrm{a}}$ avaliação recuperaram a independência funcional, com significância estatística $(p=0.000)$.

Quando se correlacionou a MIF com os dados sociodemográficos, constatou-se que a idade se correlaciona negativamente, e não influência a recuperação da independência funcional. Quanto a escolaridade, esta correlacionou de forma positiva, mas não significativa $(p=0.474)$, concluindo que quanto maior 0 nível de escolaridade maior é a recuperação da independência funcional. Relativamente ao sexo, existe uma correlação negativa, não significativa $(\mathrm{p}=0.872)$, podendo-se afirmar que os participantes do sexo masculino recuperam mais. Quanto ao estado civil, este correlacionou de forma negativa $(p=0.800)$, sendo que os participantes casados ou em união de fato recuperam mais, do que os solteiros, viúvos ou divorciados (Tabela $3)$.

Tabela 3 - Distribuição da significância entre as caraterísticas sociodemográficas e a MIF. Viana do Castelo, Portugal. $(\mathrm{n}=40)$

\begin{tabular}{|ccccccccc|}
\hline & \multicolumn{3}{c}{ Idade } & \multicolumn{2}{c}{ Sexo } & \multicolumn{2}{c|}{ Estado Civil } \\
& C. Spear. & Sig. & C. Spear. & Sig. & C. Spear. & Sig. & C. Spear. & Sig. \\
\hline MIF & -0.173 & $0.287^{*}$ & 0.117 & $0.474^{*}$ & -0.026 & $0.872^{*}$ & -0.041 & $0.800^{*}$ \\
\hline
\end{tabular}

*A correlação é significativa para o nível 0.05 .

Tabela 4 - distribuição da significância entre o tempo de imobilidade e o estado confusional com a MIF. Viana do Castelo, Portugal. $(n=40)$

Tempo de Imobilidade

C. Spearman

0.143

Sig.

\begin{tabular}{lll}
\hline MIF & 0.143 & $0.378^{*}$
\end{tabular}

NEECHAM

C. Pearson 0.263 Sig. $0.101^{*}$

*A correlação é significativa para o nível 0.05 .

Observou-se que quanto mais tempo de imobilidade os participantes possuíam, mais recuperaram, sendo que se correlacionam de forma positiva, mas esta relação não foi significativa $(p=0.378)$. Quanto ao estado confusional, este influencia a recuperação da independência funcional de forma positiva, mas não significativa $(p=0.101)$ (Tabela 4).

A amostra do estudo foi submetida a um programa de 
reabilitação, conforme descrito no método e relativamente à MIF da $1^{\mathrm{a}}$ para a $2^{\mathrm{a}}$ avaliação, os participantes passaram de dependência modificada, com assistência até $50 \%$ da tarefa (19-60 pontos) para uma dependência modificada, com uma assistência até 25\% (61-103 pontos), respetivamente, tendo recuperado $38,03 \%$ da sua independência funcional.

No que concerne ao estado confusional, os participantes passaram de uma confusão moderada a severa, numa primeira avaliação para não confusos na segunda avaliação, não corroborando aos dados do estudo ${ }^{20}$, em que os mesmos salientam que com 0 internamento 0 estado confusional agrava. Este estado pode ser precipitado por fatores associados à demência, deteoração do estado cognitivo anterior, devido aos próprios processos de imobilidade, portando da dependência, por desidratação moderada a severa e sepsis ${ }^{21}$.

A experiência de internação hospitalar é um período de mudanças significativas tanto para o paciente como para a sua família. Paciente com quadros confusionais na admissão, podem agravar $^{20}$. A alta incidência destes quadros, torna-se um grande desafio para toda a equipe multidisciplinar, encontrando-se dificultado o processo de tratamento ${ }^{20}$ nomeadamente os cuidados de reabilitação.

No presente estudo, verificou-se que o estado confusional não influência a recuperação da independência funcional, no entanto, quanto menos confusos os pacientes se encontram, mais recuperam a sua independência, não sendo este dado estatisticamente significativo para a amostra em estudo. Portanto, o estado confusional não influência a recuperação da independência funcional, no entanto, podemos ressaltar que essa melhoria no estado confusional do paciente, pode ser resultado da intervenção do enfermeiro de reabilitação já que este, no conteúdo das suas funções recupera e habilita, os pacientes vitimas de doença aguda ou descompensação de doença crónica, que causem déficit funcional cognitivo, motor, sensorial, cardio-respiratório, na deglutição, na eliminação urinária e fecal e na sexualidade ${ }^{21}$.

Analisando as carateristicas clínicas, verifica-se que os participantes com maior tempo de imobilidade recuperam mais independência funcional, podendo-se salientar que também estes têm menor score na avaliação da MIF, tendo, portanto, mais pontos, no total da escala, para 
recuperar. Tal como podemos constatar nos estudos ${ }^{21,22}$, os pacientes sujeitos a processos de imobilidade em uma unidade de cuidados intensivos, têm um compromisso importante no seu autocuidado, manifestando esses scores na MIF, mais baixos, tendo, portanto também mais pontos para recuperar.

Reconhece-se a importância da implementação precoce dos programas de reabilitação que permitam o regresso às capacidades físicas anteriores ao acontecimento agudo que motivou 0 internamento ${ }^{21}$. Por cada dia de inatividade essa potencialidade vai diminuíndo, o que tornará o regresso à independência, impossível ${ }^{23}$.

A internação por si só promove alterações no estado de saúde dos indivíduos, tanto físicas, como cognitivas, tal como já referido, e psicológicas, acrescentando-se que no momento da admissão existem alterações negativas significativas, comparativamente ao momento que precede a internação, salientando-se que no momento da alta existem alterações positivas, quando comparadas ao momento de admissão, contudo existem perdas importantes relativamente à pré-admissão ${ }^{25-27}$. Este acontecimento demarca-se sobretudo no paciente que permanece por longos períodos ventilado em unidades de cuidados intensivos, constituindo este um grupo importante de doentes que chegam aos serviços de medicina interna ${ }^{25,26}$. Depreende-se assim, a importância da implementação de programadas e intervenções de enfermagem de reabilitação que visem a promoção e dêem resposta às necessidades dos pacientes internados ${ }^{25}$ 27,29, tanto nos serviços de medicina interna como noutros serviços em que a funcionalidade e consequentemente a independência possa ser colocada em causa $^{28}$.

O contributo da enfermagem de reabilitação, assume assim, deveras importância. A enfermagem de reabilitação é considerada pela ordem dos enfermeiros como uma área de intervenção da enfermagem de excelência e de menção, na medida em que recupera e deseja capacitar de novo os pacientes vítimas de doença súbita ou descompensação da doença crónica, que incitem défice funcional, promovendo a maximização das habilidades funcionais das pessoas ${ }^{24}$.

Uma vez que os dados foram colhidos no mesmo serviço hospitalar, os participantes estiveram sujeitos ao mesmo número de doentes por enfermeiro, aos mesmos cuidados 
médicos, sociais, psicológicos, espirituais e de enfermagem de reabilitação. Nenhum destes participantes tiveram intervenção do técnico de fisioterapia.

As limitações do estudo prendem-se com o facto de ter sido incluído vários tipos de perfis de pacientes, quer seja por faixa etária, género, diagnóstico e histórico, clínico numa amostra reduzida e com a escassez de estudos recentes publicados nesta área, sugerindo-se, portanto, novas investigações sobre o tema, com maior número de participantes, onde seja possível fazer um estudo abrangente sobre os processos de imobilidade, em pacientes, portadores das mais diversas patologias, criando subgrupos e onde haja a intervenção do enfermeiros especialista em cuidados de reabilitação.

\section{CONCLUSÃO}

A amostra apresentava uma média de idade superior a 65 anos, mulheres, casadas e com o $1^{\circ}$ ciclo completo. As doenças cardiovasculares são a principal causa de morbilidade, levando a um decúbito de longa duração. Todas as

carateristicas sociodemográficas estudadas, assim como o estado confusional não influenciam a recuperação da independência funcional, devendo-se portanto, esta recuperação ao programa de reabilitação instituído pelo enfermeiro especialista em cuidados de reabilitação.

Apesar de não se poder afirmar que a mudança no score da escala NEECHAM, deve-se inteiramente ao programa de reabilitação instituído, porque existem outras variáveis que podem ter interferido, esta melhoria pode resultar em parte da intervenção do enfermeiro de reabilitação, já que este implementa intervenções com o objetivo de capacitar as funções motoras, sensoriais, cognitivas, cardíacas, respiratórias, deglutição e de eliminação.

\section{REFERÊNCIAS}

1. Lima AMN, Ferreira MSM, Martins MMFPS, Fernades CS. Influência dos cuidados de enfermagem de reabilitação na recuperação da independência do paciente. J Health NPEPS. 2019; 4(2):28-43.

2. Camões M, Fernandes $F$, Silva B, Rodrigues $T$, Costa $N$, Bezerra $P$. Exercício físico e qualidade de vida dos idosos: diferentes contextos sociocomportamentais. Motricidade. 2016; 12(1):96-105. 
3. Edelmuth SVCL, Sorio GN, Sprovieri FAA, Gali JC, Peron SF. Comorbilidades, intercorrências clínicas e fatores associados à mortalidade em pacientes idosos internados por fatura de quadril. Rev Bras Ortop. 2018; 53(5):543-551.

4. Instituto Nacional de Estatistica (PT). Mantém-se o agravamento do envelhecimento demográfico, em Portugal, que só tenderá a estabilizar daquia a cerca de 40 anos. Lisboa; 2017 [acesso em 2020 Mar. 31] Disponível em: https://www.ine.pt/xportal/xmain?xp id=INE\&xpgid=ine_destaques\&DESTAQ UESdest_boui=277695619\&DESTAQUES modo $=2 \& x$ lang $=p t$

5. Direção Geral de Saúde (DGS). Estratégia nacional para 0 envelhecimento ativo e saudável 2017-2025. Lisboa; 2017 [acesso em 2020 Mar. 31] Disponível em: https://www.sns.gov.pt/wpcontent/uploads/2017/07/ENEAS.pdf

6. Ordem dos Enfermeiros (OE). Classificação Internacional para a prática de Enfermagem - Versão 2015. Lisboa: Lusodidacta; 2016.

7. Ministério da Saúde (PT). Retrato da saúde. Lisboa: Ministério da Saúde; 2018.

8. Piredda M, Bartiromo C, Capuzzo MT,
Matarese M, Marinis MG. Nursing care dependence in the experiences of advanced cancer impatientes. Eur J Oncol Nurs. 2016; 20(2):125-132.

9. Phipps W. Enfermagem médicocirúrgica - conceitos e prática clínica. Loures: Lusociência; 2009.

10. Carrilho L, Gameiro C, Ribeiro A. Envelhecer no concelho de Oeiras: Estudo numa população institucionalizada. Anál Psicol. 2015; 1(35):121-135.

11. Fontoni MR, Oliveira WL, Kaneta CN. Winnicotti e o desafio do atendimento a pacientes idosos em estado confusional. Psicol saúde doenças. 2014; 15(3): 816-827.

12. Ordem dos Enfermeiros (PT). Cuidados à Pessoa com Alterações da Mobilidade - Posicionamentos, Transferências e Treino de Deambulação. Lisboa: Ordem dos Enfermeiros; 2013.

13. Ministério da Saúde (PT). Medicina Física e de Reabilitação. Lisboa; 2017 [acesso em 2020 Mar. 31]. Disponível em: https://www.sns.gov.pt/wpcontent/uploads/2017/03/2017-01 27-

RNEHRMedFisicaReabVersaoFinal.pdf 14. Laíns J. Sistema uniformizado de dados para reabilitação médica. 
Coimbra; 1991 [acesso em 2019 set. 25]. Disponível em: http://rihuc.huc.minsaude.pt/handle/10400.4/541

15. Prado ARA, Ramos RL, Ribeiro OMPL, Figueiredo NMA, Martins MM, Machado WCA. Bath for dependent patients: theorizing aspects of nursing care in rehabilitation. Rev Bras Enferm. 2017; 70(6):1337-42.

16. Diário da República (PT). Regulamento das competências especificas do enfermeiro especialista em Enfermagem de Reabilitação. Lisboa; 2019 [acesso em 2020 Mar. 31]. Disponível em: https://dre.pt/application/conteudo /122216893

17. Base de Dados Portugal Contemporâneo. Esperança de vida a nascença: total e por sexo (base: triennio a partir de 2001). Lisboa; 2019 [acesso em 2019 out. 26]. Disponivel em: https://www.pordata.pt/Portugal

18. Neves $H$, Silva $A$, Marques $P$. Tradução e adapatação cultural da escala de confusão de NEECHAM. Rev Enferm Ref. 2011; 3(3):105-112.

19. Riberto M, Miyazaki MH, Jucá SSH, Sakamoto H, Pinto PPN, Battistella LR. Validação da Versão Brasileira da Medida de Independência Funcional. Acta fisiátrica. 2004; 11(2):72-76.
20. Fontoni MR, Oliveira WL, Kaneta CN. Winnicitt and the challeng in care of elderly patients with confusional state. Psic saúde doenças. 2014; 15(3):816-827.

21. Ordem dos Enfermeiros. Padrões de qualidade dos cuidados especializados em enfermagem de reabilitação. Lisboa; 2018 [acesso em 2020 mar. 15]. Disponível em: https: / /www.ordemenfermeiros.pt/ media/8141/ponto-4_regulamentodos-padr\%C3\%B5es-qualidadeceer.pdf

22. Tineo JPA, Ascencio LAC, Moya CRR, Caballero SP, Aguilar CAC, Alva RV. Factores associados a síndrome confusional agudo em adultos mayores internados em emergência de um hospital terciário. An Fac Med. 2013; 74(3):193-7.

23. Sistema Nacional de Saúde. Rede Nacional de Especialidade Hospitalar e de Referenciação - Medicina Física de Reabilitação. Lisboa; 2017 [acesso em 2020 mar. 15]. Disponível em: https://www.sns.gov.pt/wpcontent/uploads/2017/03/2017-0127-

RNEHRMedFisicaReabVersaoFinal.pdf 24. Ordem dos Enfermeiros. Regulamento dos padrões de qualidade dos cuidados 
especializados em enfermagem de reabilitação. Lisboa: Ordem dos Enfermeiros; 2011.

25. Azevedo PMDS, Gomes BP, Pereira JATP, Carvalho FMN, Ferreira SP, Pires Al, et al. Functional dependence at discharge from the intensive care unit, relevance for rehabilitation nursing. Rev Enferm Ref. 2019; 5(20):37-46.

26. Pereira DM, Tamaki EM, Andrade SMO, Rapello VG, Reis FA, Junior SCS. Functional Independence of elderly patients who received invasive mechanical ventilation: importance for the clinical outcome and effects of hospitalization. Conscientiae saúde. 2018; 17(2):135-
27. Dornelles SQ, Signori LU, Corrêa LQ, Silveira DF, Guerreiro LF, Teixeira AO. Effects of hospitalization on functional capacity and quality of life of cardimetabolic patients. Conscientiae saúde. 2014; 13(1):101109.

28. Ganefianty A, Yona S. A nursing rehabilitation program to improve the quality of life of patients with meningioma: A narrative review. Belitung nurs j. 2019; 5(2):60-64.

29. Azevedo PMDS, Gomes BP. Effects of early in the functional rehabilitation of critically ill patients: a systematic review. Rev Enferm Ref. 2015; 5:129-138.

143.

Financiamento: Os autores declaram que não houve financiamento.

Conflito de interesses: Os autores declaram não haver conflito de interesses.

Participação dos autores:

- Concepção: Lima AMN, Ferreira MSM, Martins MMFPS, Fernandes CS, Moreira MTF, Rodrigues TMP

- Desenvolvimento: Lima AMN, Ferreira MSM, Martins MMFPS, Fernandes CS, Moreira MTF, Rodrigues TMP.

- Redação e revisão: Lima AMN, Ferreira MSM, Martins MMFPS, Fernandes CS, Moreira MTF, Rodrigues TMP.

Como citar este artigo: Lima AMN, Ferreira MSM, Martins MMFPS, Fernandes CS, Moreira MTF, Rodrigues TMP. Independência funcional e o estado confusional de pessoas sujeitas a programa de reabilitação. J Health NPEPS. 2020; 5(2):XXX-XXX. 